\title{
Three-Dimensional Holographic Imaging Using Single Frequency Microwave Data
}

\author{
Reza K. Amineh (D), Maryam Ravan (D), Raveena Sharma (D), and Smit Baua $\mathbb{D}^{D}$ \\ Department of Electrical and Computer Engineering, New York Institute of Technology, New York, NY, USA \\ Correspondence should be addressed to Reza K. Amineh; rkhalaja@nyit.edu
}

Received 30 March 2018; Revised 2 June 2018; Accepted 14 June 2018; Published 17 July 2018

Academic Editor: Ahmed Toaha Mobashsher

Copyright ( 2018 Reza K. Amineh et al. This is an open access article distributed under the Creative Commons Attribution License, which permits unrestricted use, distribution, and reproduction in any medium, provided the original work is properly cited.

\begin{abstract}
Three-dimensional (3D) microwave and millimeter wave imaging techniques based on the holographic principles have been successfully employed in several applications such as security screening, body shape measurement for the apparel industry, underground imaging, and wall imaging. The previously proposed 3D holographic imaging techniques require the acquisition of wideband data over rectangular or cylindrical apertures. Requirement for wideband data imposes limitations on the hardware (in particular at very high or very low frequencies). It may also lead to errors in the produced images if the media is dispersive (e.g., in biomedical imaging) and not modeled properly in the image reconstruction process. To address these limitations, here, we propose a technique to perform 3D imaging with single frequency data. Instead of collecting data at multiple frequencies, we acquire the backscattered fields with an array of resonant antennas. We demonstrate the possibility of 3D imaging with the proposed setup and perform a comprehensive study of the capabilities and limitations of the technique via simulations. To perform a realistic study, the simulation data is contaminated by noise.
\end{abstract}

\section{Introduction}

One of the most practical and promising three-dimensional (3D) microwave and millimeter wave imaging approaches is based on the modern holographic concepts.

Holographic-based imaging concept was originally introduced in the field of optical imaging when Gabor $[1,2]$ aimed at improving the images obtained by an electron microscope, but he demonstrated the feasibility of his method with light waves. For this purpose, he demonstrated the possibility of acquiring the magnitude and phase of a wave as an interference pattern formed by this wave and a known reference wave. Gabor's experimental setup was improved by Leith and Upatnieks $[3,4]$ in the early sixties to achieve higher quality images. The work of Leith and Upatnieks resonated well with the availability of lasers leading to a new generation of 3D imaging systems. The progress in the field of optical holography inspired researchers in other fields, in particular acoustics [5] and microwave [6-10], to aim at the image reconstruction of optically opaque objects. Thus, in this early stage, microwave holography closely resembled the optical method for data acquisition referred to as the "recording step" and that for the image reconstruction referred to as "reconstruction step" [11, 12]. In the recording step, an intensity pattern (hologram) is formed from the interference pattern between the scattered wavefront and a coherent plane wave, introduced at an offset angle to the recording plane. This intensity pattern is acquired through the scanning of an antenna over the acquisition plane, the antenna being connected to a simple diode detector (a receiver that measures only the signal intensity) [13].

The subsequent progress in the field of holographic microwave and millimeter wave imaging (MMI) can be divided into two separate paths: indirect holography and direct holography. What is common between these two categories of techniques is that both exploit the magnitude and the phase information of the backscattered waves in order to generate images and to achieve resolution enhancement compared to the images obtained from raw amplitude measurements only.

The indirect microwave holography has been in fact the extension of the early works on this topic which has the most resemblance to optical holography in terms of recording a hologram. The major advantage of indirect holography is 
that it does not require the use of expensive vector measurement equipment to obtain the complex field scattered from the imaged object. Instead, the phase information is mathematically recovered from low-cost intensity-only scalar microwave or millimeter wave measurements. This simplifies the hardware implementation and reduces the cost of the imaging system (e.g., see [14-16]).

The direct holographic MMI techniques, however, use radio frequency (RF) circuitry or vector network analyzer (VNA) to record both the magnitude and phase of the backscattered waves directly (e.g., see [17-18]). Although, similar to recording a hologram in indirect holography, measurements are typically performed over an aperture (plane); the scattered waves are sampled in complex form (as opposed to intensity-only measurements in indirect holography). As the direct holographic algorithms developed, their resemblance to synthetic aperture radar- (SAR-) based imaging methods became evident. In both, the complex-valued backscattered waves are acquired over an aperture. It has been shown in [19] that by collecting wideband information over a linear aperture, two-dimensional (2D) imaging is possible along cross-range and range directions. This algorithm was developed further at the Pacific Northwest National Laboratory (PNNL) [17]. There, 3D imaging has been accomplished by acquiring wideband data over a rectangular aperture. Later, this approach was extended further to perform 3D imaging by data acquisition over a cylindrical aperture [18]. At PNNL, these techniques have been successfully used for practical imaging of human body's surface with applications in threat detection (for security screening) [17, 18], body shape measurement for the apparel industry [20], and imaging of underground objects and objects hidden inside the cement walls [21].

The processing in $[17,18,20,21]$ relies on an assumed analytical (exponential) form for the incident and the Green's functions to cast the inversion in the form of a 3D inverse Fourier transform (FT). Despite being fast and robust, the abovementioned holographic techniques suffer the following drawbacks:

(1) Far-field assumptions (assuming analytic forms for the incident field and Green's function) limit the performance of these techniques to far-zone imaging. Imaging errors caused by far-field assumptions might be less noticeable for large and high contrast objects such as those imaged in [22]. In those cases, it is still possible to reconstruct satisfactory qualitative images. However, these errors can be noticed very well in more challenging imaging scenarios for smaller and lower contrast objects. This has been demonstrated in [23].

(2) The wave numbers $k_{x}, k_{y}$, and $k_{z}$ are assumed independent variables, and this leads to errors in the image reconstruction process.

(3) Resampling of the data in $k_{z}$ space is necessary. This further degrades the quality of the reconstructed images.
(4) Since these techniques have been developed based on the far-field assumptions, they are incapable of processing the evanescent waves. However, these waves can be partially measured in the nearfield imaging applications to provide higher crossrange resolutions.

(5) The transmitting and receiving antennas are assumed to be pointwise.

(6) They have been developed based on the measurement of the backscattered waves of a single antenna.

To address the limitations of the techniques in $[17,18]$, the direct holography technique was extended first to near-field 2D imaging [24] and then to 3D imaging $[23,25]$. In $[23,25]$, the analytical approximations of the incident field and the Green's function are inadequate; that is why numerical models are employed instead, which better represent the particular acquisition setup and antennas. These methods also allow for incorporating forwardscattered signals in addition to the backscattered signals acquired on two opposite rectangular apertures. This is in particular important in biomedical imaging since, there, the forward-scattered waves have much higher SNR compared to the backscattered waves. Due to that, in [26], the 3D holographic techniques in $[23,25]$ was extended for the single transmitter and multiple receivers and only forwardscattered data was employed to reconstruct 3D images of the inspected medium. The processing in $[23,25,26]$ involves the solution of a linear system of equations for each spatial frequency pair $\left(k_{x}, k_{y}\right)$. Then, 2D inverse FT is applied to the solution on planes (slices) at all desired range locations. These linear systems of equations have much smaller dimensions and are less ill-conditioned compared to the systems of equations arising in optimization-based imaging techniques. Furthermore, the resampling of the data in $k_{z}$ space is avoided. The assumption that $k_{x}, k_{y}$, and $k_{z}$ are independent variables is also irrelevant since the use of parameter $k_{z}$ is avoided.

In the near-field holographic imaging techniques proposed in [23-26], the data for the incident field and the Green's function are obtained via simulations. In practice, often, the fidelity of the simulation models, although better than the analytical approximations, may still be too low to ensure good image quality. To resolve this issue, in [27], a method has been proposed to acquire the incident field and the Green's function specific to the particular acquisition system via measurements of a known calibration object (CO). The method exploits the concept of point spread function (PSF) of a linear imaging system where the response due to an arbitrary object is the convolution of the response due to a pointwise scatterer (the scattering probe) with the spatial variation of that object. This approach provides a fast and robust means to reconstruct qualitative images of the objects.

Although the abovementioned near-field holographic microwave imaging algorithms can provide real-time 3D images of the dielectric media, the following drawbacks still need to be addressed before they can be employed in a compact, portable, and low-cost imaging setup: 
(1) Since these techniques require wideband data collection, the data acquisition system including antennas and circuitry become complex, costly, and bulky.

(2) Implementation of the compact and low-cost data acquisition techniques such as modulated scatterer technique (MST) is not straightforward and efficient for wideband systems. These data acquisition techniques allow for using electronic scanning instead of mechanical scanning of the sensor over the aperture. Thus, in a single frequency imaging system, they can drastically reduce the data acquisition time paving the way toward real-time imaging applications.

(3) Due to the use of wideband data, dispersive properties of the media which may not be modeled accurately impose additional errors on the reconstructed images.

(4) In a wideband data acquisition system, measuring scattering $(S)$ parameters by frequency sweep takes time while measurement time is critical in many applications such as object tracking or medical imaging (patient movement during a scan may generate artifacts).

Although, in [28], single frequency 3D microwave holographic imaging has been proposed but it is based on the far-field approximations. All the previous 3D near-field holographic imaging works are based on the wideband data and thus they suffer the drawbacks enumerated above. To address the abovementioned drawbacks, here, we demonstrate the feasibility of performing 3D holographic imaging using single frequency data. For this purpose, an array of resonant antennas are employed to perform data acquisition. The data is then processed with the holographic image reconstruction algorithm. Simulations are employed to study various parameters such as the number of antennas, number of reconstruction lines (planes), distance between reconstruction lines (planes), and effect of noise. To perform a realistic study, simulation data is contaminated by noise.

\section{Background: 3D Holographic Imaging Using Wideband Microwave Data [27]}

Figure 1(a) illustrates a wideband microwave holographic imaging setup including a transmitter antenna that illuminates the object while moving over a rectangular aperture. The same antenna or a receiver antenna that moves together with the transmitter antenna is employed to scan the backscattered field over the aperture. At each sampling position $(x, y)$, the complex-valued scattered wave due to the object $E^{s c}(x, y, f)$ is measured at $N_{f}$ frequencies. The objective is to reconstruct images over $z=z_{i}$ planes where $i=1, \ldots, N_{z}$.

Here, the imaging system is assumed to be linear. It is well-known that the use of Born approximation for the scattering integral leads to the linear property of the imaging system (e.g., see [29]). This approximation indicates that the field inside the object is approximated with the field in the absence of the object. In addition to the linear property, the imaging system is assumed to be spaceinvariant, that is, if an object is shifted in a plane parallel to the $x-y$ plane in Figure 1, its response will be shifted by the same amount and along the same direction. Assuming that the imaging system is both linear and space-invariant allows for the use of convolution theory as it will be discussed briefly in the following.

According to the convolution theory, in a linear spaceinvariant system, the response to any arbitrary input function to the system can be written in terms of the convolution of the point spread function (PSF) of the system and that input function. PSF of the system is actually the response of the system to a Dirac delta function. This concept has been used in [27] to perform holographic imaging of dielectric media. In order to collect the responses corresponding to PSFs, we first record the responses $E_{i}^{\text {sc,co }}$ due to pointwise scatterers placed at $\left(0,0, z_{i}\right), i=1, \ldots, N_{z}$, as illustrated in Figure $1(\mathrm{~b})$. Similar to [27], we refer to the pointwise scatterers as calibration objects (COs). These are the smallest objects that can be measured at $\left(0,0, z_{i}\right), i=1, \ldots, N_{z}$ positions. Responses measured for such small objects approximate the responses of the system to Dirac delta input functions. These COs are placed one at a time at each imaged plane at $\left(0,0, z_{i}\right), i=1, \ldots$, $N_{z}$ positions while the scattered response due to each CO $E_{i}^{s c, c o}(x, y, f)$ is measured over the aperture by scanning the transmitter-receiver antennas. As we described, these responses provide approximations of the PSFs for this linear space-invariant imaging system. Thus, the response $E_{i}^{\mathrm{sc}}(x, y)$ due to an arbitrary object at the plane $z=z_{i}$ can be obtained by the convolution of the collected PSF for the corresponding plane with the contrast function $c_{i}(x, y)$ due to the presence of the object (contrast between the electrical properties of the object and the background medium) on that plane. This is written as

$$
E_{i}^{\mathrm{sc}}(x, y)=E_{i}^{\mathrm{sc}, \mathrm{co}}(x, y) *_{x} *_{y} c_{i}(x, y)
$$

where $*_{x}$ and $*_{y}$ denote convolutions with respect to the $x$ and $y$ variables, respectively. Equation (1) can be written for responses created by the unknown objects over each imaged plane $\left(0,0, z_{i}\right), i=1, \ldots, N_{z}$. Then, the total response $E^{\mathrm{sc}}(x, y)$ measured by the receiver antenna due to the presence of the objects at all imaged planes can be approximated with the superposition of the contribution of all these responses as

$$
E^{\mathrm{sc}}(x, y)=\sum_{i=1}^{N_{z}} E_{i}^{\mathrm{sc}}(x, y)=\sum_{i=1}^{N_{z}} E_{i}^{\mathrm{sc}, \mathrm{co}}(x, y) *{ }_{x} *_{y} c_{i}(x, y) .
$$

In (1), $E_{i}^{\mathrm{sc}, \mathrm{co}}(x, y)$ functions are known due to the measurement or simulation of the responses to COs. $E_{i}^{\mathrm{sc}}(x, y)$ is also known due to the recording of the response for the unknown object. In order to estimate the unknown contrast functions $c_{i}(x, y)$ on the imaged planes $z=z_{i}, i=$ $1, \ldots, N_{z}$, the scattered data is acquired at multiple frequencies, $f_{n}, n=1, \ldots, N_{f}$. Thus, (2) can be rewritten at all these measurement frequencies to provide the following system of equations: 


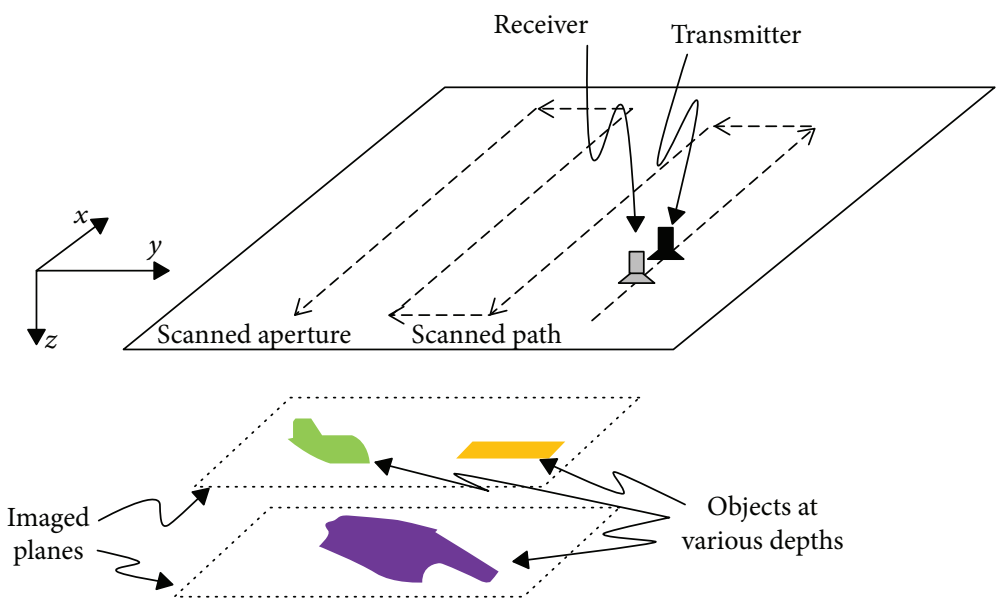

(a)

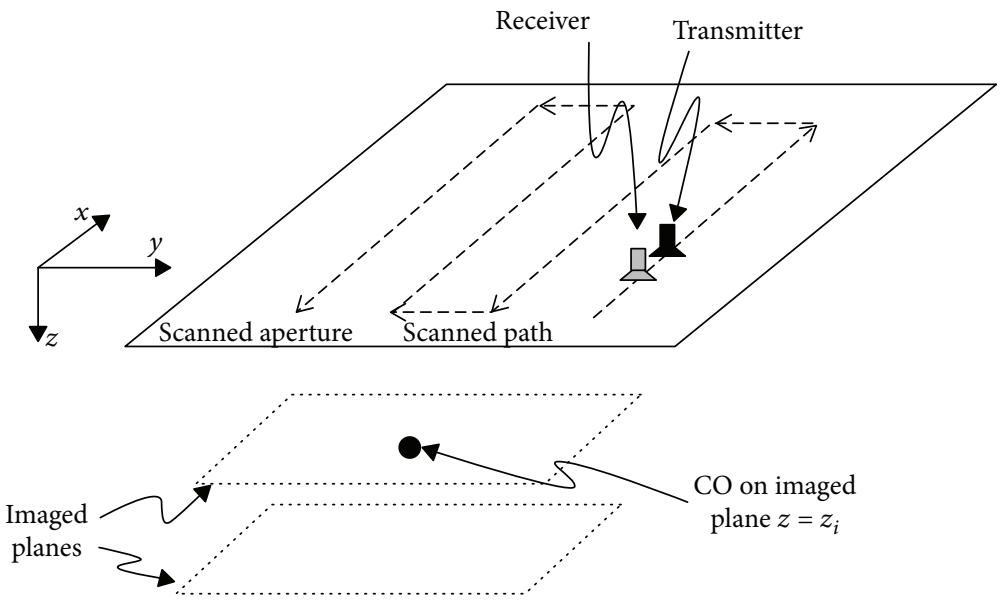

(b)

FIGURE 1: (a) Illustration of a wideband 3D microwave holographic imaging setup that consists of a transmitter antenna and a receiver antenna that move together to scan a rectangular aperture. (b) Illustration of placing a CO on the imaged plane $z=z_{i}$ to measure the PSF corresponding to that plane, that is, $E_{z_{i}}^{\mathrm{sc}, \mathrm{co}}(x, y, f)$.

$$
\begin{gathered}
E^{\mathrm{sc}}\left(x, y, f_{1}\right)=\sum_{i=1}^{N_{z}} E_{i}^{\mathrm{sc}, \mathrm{co}}\left(x, y, f_{1}\right) *_{x} *_{y} c_{i}(x, y), \\
\vdots \\
E^{\mathrm{sc}}\left(x, y, f_{N_{f}}\right)=\sum_{i=1}^{N_{z}} E_{i}^{\mathrm{sc}, \mathrm{co}}\left(x, y, f_{N_{f}}\right) *_{x} *_{y} c_{i}(x, y) .
\end{gathered}
$$

In order to solve the system of equation in (3), 2D FTs with respect to $x$ and $y$ variables are applied on both sides of the equations. This leads to the following system of equations in the Fourier domain:

$$
\begin{gathered}
\tilde{\widetilde{E}}^{\mathrm{sc}}\left(k_{x}, k_{y}, f_{1}\right)=\sum_{i=1}^{N_{z}} \tilde{\widetilde{E}}_{i}^{\mathrm{sc}, \mathrm{co}}\left(k_{x}, k_{y}, f_{1}\right) \tilde{\tilde{c}}_{i}\left(k_{x}, k_{y}\right), \\
\vdots \\
\tilde{\tilde{E}}^{\mathrm{sc}}\left(k_{x}, k_{y}, f_{N_{f}}\right)=\sum_{i=1}^{N_{z}} \tilde{\tilde{E}}_{i}^{\mathrm{sc}, \mathrm{co}}\left(k_{x}, k_{y}, f_{N_{f}}\right) \tilde{\tilde{c}}_{i}\left(k_{x}, k_{y}\right),
\end{gathered}
$$

where $\tilde{\widetilde{E}}^{\mathrm{sc}}\left(k_{x}, k_{y}, f_{n}\right), \tilde{\tilde{E}}_{i}^{\mathrm{sc}, \mathrm{co}}\left(k_{x}, k_{y}, f_{n}\right)$, and $\tilde{\tilde{c}}_{i}\left(k_{x}, k_{y}\right)$ are the 2D FTs of the functions $E^{\mathrm{sc}}\left(x, y, f_{n}\right), E_{i}^{\mathrm{sc}, \mathrm{co}}\left(x, y, f_{n}\right),(n=1$, $\left.\ldots, N_{f}\right)$, and $c_{i}(x, y)$, respectively, and $k_{x}$ and $k_{y}$ are Fourier variables corresponding to the $x$ and $y$ variables, respectively. The system of equations in (4), written for each spatial frequency pair $\left(k_{x}, k_{y}\right)$, is solved in a least square sense to find $\tilde{\tilde{c}}_{i}\left(k_{x}, k_{y}\right), i=1, \ldots, N_{z}$. Due to the relatively small size of the system of equations in (4), the method usually yields well-conditioned matrices. Yet, well-conditioned matrices do not always ensure good quality images. When the SNR is poor, the image quality can be improved by low-pass filtering or regularization, for example, the Tikhonov regularization [30]. Once the systems of equations are solved for all $\left(k_{x}, k_{y}\right)$, the inverse $2 \mathrm{D}$ FT is applied to $\tilde{\tilde{c}}_{i}\left(k_{x}, k_{y}\right), i=1, \ldots$, $N_{z}$, to reconstruct a $2 \mathrm{D}$ slice of the contrast function $c_{i}$ $(x, y)$ at each $z=z_{i}$ plane. Then, the normalized modulus of $c_{i}(x, y),\left|c_{i}(x, y)\right| / M$, where $M$ is the maximum of $\left|c_{i}(x, y)\right|$ for all $z_{i}$, is plotted versus $x$ and $y$ to obtain 2D images of the object at all $N_{z}$ planes. By putting together all 2D slice images, a $3 \mathrm{D}$ image of the object is obtained. 


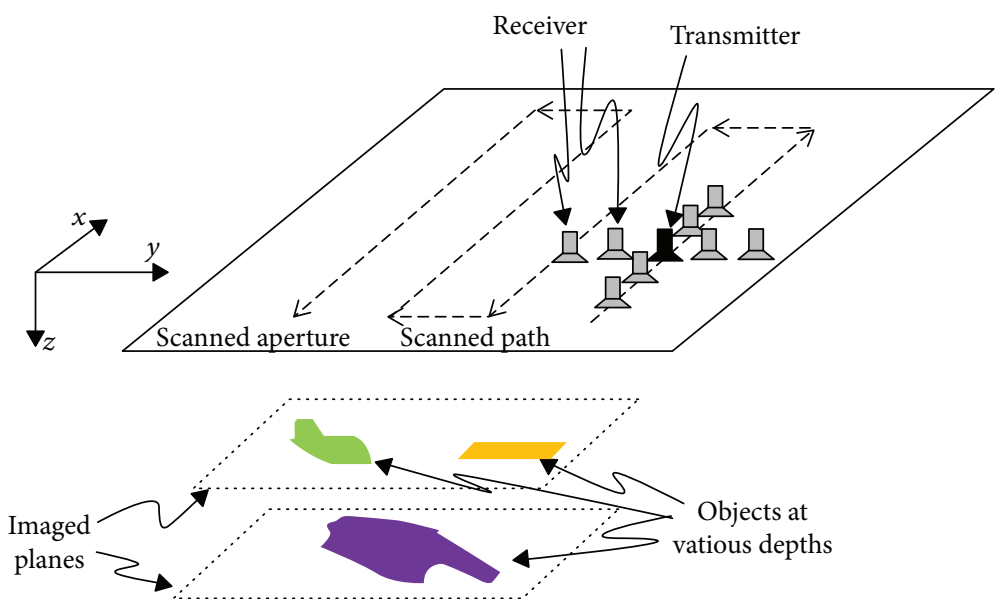

FIGURE 2: Illustration of the single frequency 3D holographic imaging setup which contains a transmitting antenna that illuminates the inspected medium with microwave power at a single frequency and when moving with an array of $N_{r}$ receiver antennas. This transmitterreceiver set scans a rectangular aperture.

\section{Proposing Holographic Imaging with Single Frequency Microwave Data}

In the previous section, we described that in order to solve (2) and find the contrast functions $c_{i}(x, y)$ over all reconstruction planes $z=z_{i}, i=1, \ldots, N_{z}$, in [27], measurements are performed at multiple frequencies $f_{n}, n=1, \ldots, N_{f}$. That enables forming the system of equation in (3) which then can be solved to find the contrast functions $c_{i}(x, y), i=1, \ldots, N_{z}$. In this section, we propose using $N_{r}$ receivers that sample the scattered field $E_{n_{r}}^{\mathrm{sc}}(x, y), n_{r}=1, \ldots, N_{r}$ due to the object at spatially diverse positions for each scanning position of the transmitter. Figure 2 illustrates this configuration. Similar to using multiple frequency data, using multiple receiver data allows for rewriting (2) for each receiver and constructing a system of equations as follows:

$$
\begin{gathered}
E_{1}^{\mathrm{sc}}(x, y)=\sum_{i=1}^{N_{z}} E_{1, i}^{\mathrm{sc}, \mathrm{co}}(x, y) *_{x} *_{y} c_{i}(x, y), \\
\vdots \\
E_{N_{r}}^{\mathrm{sc}}(x, y)=\sum_{i=1}^{N_{z}} E_{N_{r}, i}^{\mathrm{sc}, \mathrm{co}}(x, y) *_{x} *_{y} c_{i}(x, y),
\end{gathered}
$$

where $E_{n_{r}, i}^{\mathrm{scc}, i}(x, y), n_{r}=1, \ldots, N_{r}$, is the measured or simulated PSF recorded by the receiver $n_{r}$ and when the CO is placed at imaged plane $z=z_{i}$. The array of receivers can be on the same side of the inspected medium as the transmitter antenna or on the opposite side of that. Solution of this system of equations is similar to what we discussed above for the wideband imaging system. 2D FTs with respect to the $x$ and $y$ variables are applied on both sides of (5). This leads to the following system of equations:

$$
\begin{gathered}
\tilde{\tilde{E}}_{1}^{\mathrm{sc}}\left(k_{x}, k_{y}\right)=\sum_{i=1}^{N_{z}} \tilde{\tilde{E}}_{1, i}^{\mathrm{sc}, \mathrm{co}}\left(k_{x}, k_{y}\right) \tilde{\tilde{c}}_{i}\left(k_{x}, k_{y}\right), \\
\vdots \\
\tilde{\tilde{E}}_{N_{r}}^{\mathrm{sc}}\left(k_{x}, k_{y}\right)=\sum_{i=1}^{N_{z}} \tilde{\tilde{E}}_{N_{r}, i}^{\mathrm{sc}, \mathrm{co}}\left(k_{x}, k_{y}\right) \tilde{\tilde{c}}_{i}\left(k_{x}, k_{y}\right),
\end{gathered}
$$

where $\tilde{\widetilde{E}}_{n_{r}}^{\mathrm{sc}}\left(k_{x}, k_{y}\right)$ and $\tilde{\tilde{E}}_{n_{r}, i}^{\mathrm{sc}, \mathrm{co}}\left(k_{x}, k_{y}\right)$ are the 2D FTs of the functions $E_{n_{r}}^{\mathrm{sc}}(x, y)$ and $E_{n_{r}, i}^{\mathrm{sc}, \mathrm{co}}(x, y)$, respectively. In the following section, we study the number of antennas that are required to obtain range resolution when collecting data only at a single frequency.

To ensure processing the useful portion of the spectrum corresponding to the measurable values of $k_{x}$ and $k_{y}$, we consider the maximum values of these Fourier variables to be

$$
\begin{aligned}
& k_{x}^{\max }=2 k \sin \frac{\theta_{b, x}}{2}, \\
& k_{y}^{\max }=2 k \sin \frac{\theta_{b, y}}{2},
\end{aligned}
$$

where $k$ is the wavenumber in the background medium, and $\theta_{b, x}$ and $\theta_{b, y}$ are the lesser of the full beam width of the antenna or the angle subtended by the aperture along the $x$ and $y$ directions, respectively [17]. This results in the crossrange resolutions of

$$
\begin{aligned}
& \delta_{x}=\frac{\lambda}{4 \sin \left(\theta_{b, x} / 2\right)}, \\
& \delta_{y}=\frac{\lambda}{4 \sin \left(\theta_{b, y} / 2\right)} .
\end{aligned}
$$

Since, in general, $\theta_{b, x}$ and $\theta_{b, y}$ could be different along $x$ or $y$ directions, the resolutions for these two cross-range directions can be different. 


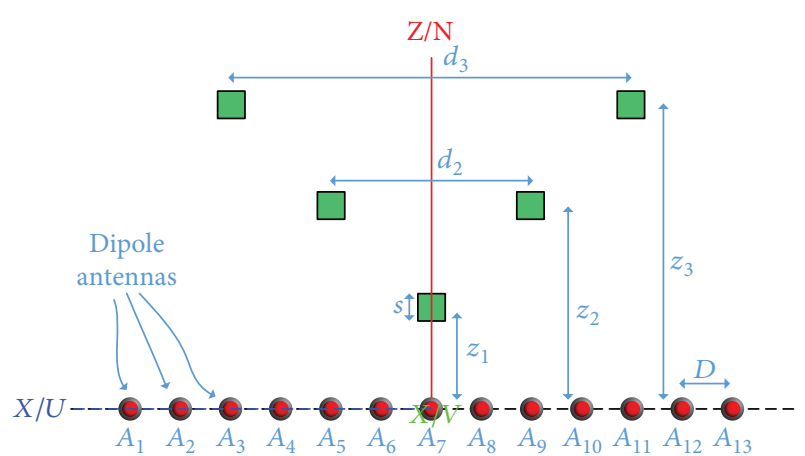

Figure 3: Cross-section view for the imaging setup in FEKO software.

\section{Image Reconstruction Results}

In order to study the performance of the proposed imaging technique, we conduct simulation study using FEKO software [31]. The study is performed at operation frequency of $6.5 \mathrm{GHz}$. However, all the dimensions are reported in terms of wavelength $\lambda$ so that the outcome of the study can be readily extended to other frequencies. To understand the capabilities and limitations of the proposed 3D imaging technique, first $2 \mathrm{D}$ imaging results along one cross-range direction $(x$-axis) and range direction $(z$-axis) are presented and discussed. Then imaging results for $3 \mathrm{D}$ imaging examples will be presented.

4.1. Studying the Feasibility of Achieving Range Resolution with $2 D$ Imaging Results. Figure 3 shows the proposed imaging setup to perform $2 \mathrm{D}$ imaging in which an array of resonant $(\lambda / 2)$ dipole antennas is employed along the $x$-axis to acquire the backscattered data from objects placed at different positions. The background medium has properties of $\varepsilon_{r}=16$ and $\sigma=0.5 \mathrm{~S} / \mathrm{m}$. The objects are cubes of identical size with the side dimension denoted by parameter $s$, and they all have properties $\varepsilon_{r}=32$ and $\sigma=1 \mathrm{~S} / \mathrm{m}$. The interelement spacing for the antenna array is denoted by $D$. The images are reconstructed on multiple lines along the $x$-axis and placed at $z=z_{i}, i=1, \ldots, N_{z}$. The distance between the objects on line $z=z_{i}$ is denoted by $d_{i}$. To have a realistic study, all the image reconstruction results are presented with considering an additive White Gaussian noise with signal-to-noise ratio (SNR) of $20 \mathrm{~dB}$ that contaminates the simulated data. However, in the case that we perform the study of effect of SNR on the images, the SNR value will be lowered.

In the first example, with reference to Figure 3, an array of 13 resonant dipole antennas is employed for performing imaging on three lines at $z_{1}=0.5 \lambda, z_{2}=\lambda$, and $z_{3}=1.5 \lambda$ (distance between image lines is $\lambda / 2$ ). The distances between the objects are $d_{2}=\lambda$ and $d_{3}=2 \lambda$. The interelement spacing for the array of dipoles is $D=0.25 \lambda$. An aperture of size $14 \lambda$ is scanned by this array of antennas, that is, the center element $A_{7}$ in Figure 3 scans from $-7 \lambda$ to $7 \lambda$. At each sampling position, $A_{7}$ is excited while all the antennas including $A_{7}$ record the backscattered data due to
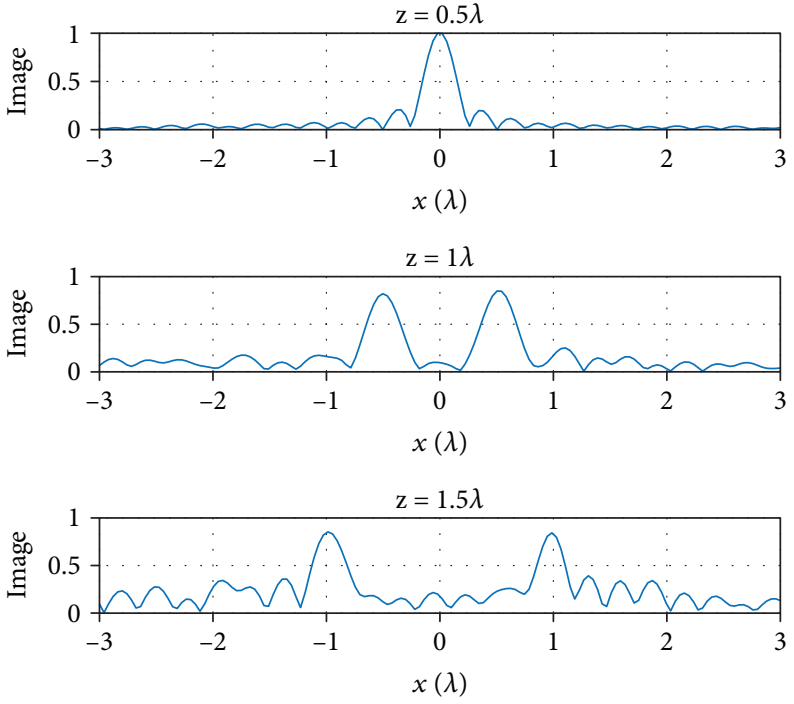

FIgURE 4: Reconstructed images when using 13 resonant dipole antennas. For this example, the parameters are $z_{1}=0.5 \lambda, z_{2}=\lambda$, $z_{3}=1.5 \lambda, d_{2}=\lambda, d_{3}=2 \lambda, s=\lambda / 8$, and $D=0.25 \lambda$. These results are with $\mathrm{SNR}=20 \mathrm{~dB}$.

the objects. To implement the holographic imaging technique, PSF functions are recorded beforehand by sequentially putting small cubes with side of $\lambda / 8$ at $(0,0,0.5 \lambda),(0,0, \lambda)$, and $(0,0,1.5 \lambda)$ positions and for each case, the backscattered waves due to these objects are scanned by the antenna array. Figure 4 shows the reconstructed images. It is observed that the objects are reconstructed well at their true positions.

Next, to study the effect of a number of antennas on the reconstructed images, an array of 9 resonant dipole antennas is employed for imaging. The setup and other parameters in Figure 3 are the same as those mentioned for the first example. At each sampling position, the center element, that is, $A_{5}$ for 9 antennas, is excited while all the antennas record the backscattered data due to the objects.

Figure 5 shows the reconstructed images. By comparing the results in Figures 4 and 5, it is clearly observed that reducing the number of antennas degrades the quality of the images.

It has been discussed in [32] that if the contrast function has only real part (e.g., for lossless media), then the 2D FT of that would be a Hermitian function, that is,

$$
\tilde{\tilde{c}}_{i}\left(-k_{x},-k_{y}\right)=\tilde{\tilde{c}}_{i}^{*}\left(k_{x}, k_{y}\right)
$$

where $(.)^{*}$ is the complex conjugate operator. Using (9) and (6), another system of equations can be built as

$$
\begin{gathered}
\tilde{\tilde{E}}_{1}^{\mathrm{sc} *}\left(-k_{x},-k_{y}\right)=\sum_{i=1}^{N_{z}} \tilde{\tilde{E}}_{1, i}^{\mathrm{sc}, \mathrm{co} *}\left(-k_{x},-k_{y}\right) \tilde{\tilde{c}}_{i}\left(k_{x}, k_{y}\right), \\
\vdots \\
\tilde{\tilde{E}}_{N_{r}}^{\mathrm{sc} *}\left(-k_{x},-k_{y}\right)=\sum_{i=1}^{N_{z}} \tilde{\tilde{E}}_{N_{r}, i}^{\mathrm{sccco} *}\left(-k_{x},-k_{y}\right) \tilde{\tilde{c}}_{i}\left(k_{x}, k_{y}\right) .
\end{gathered}
$$



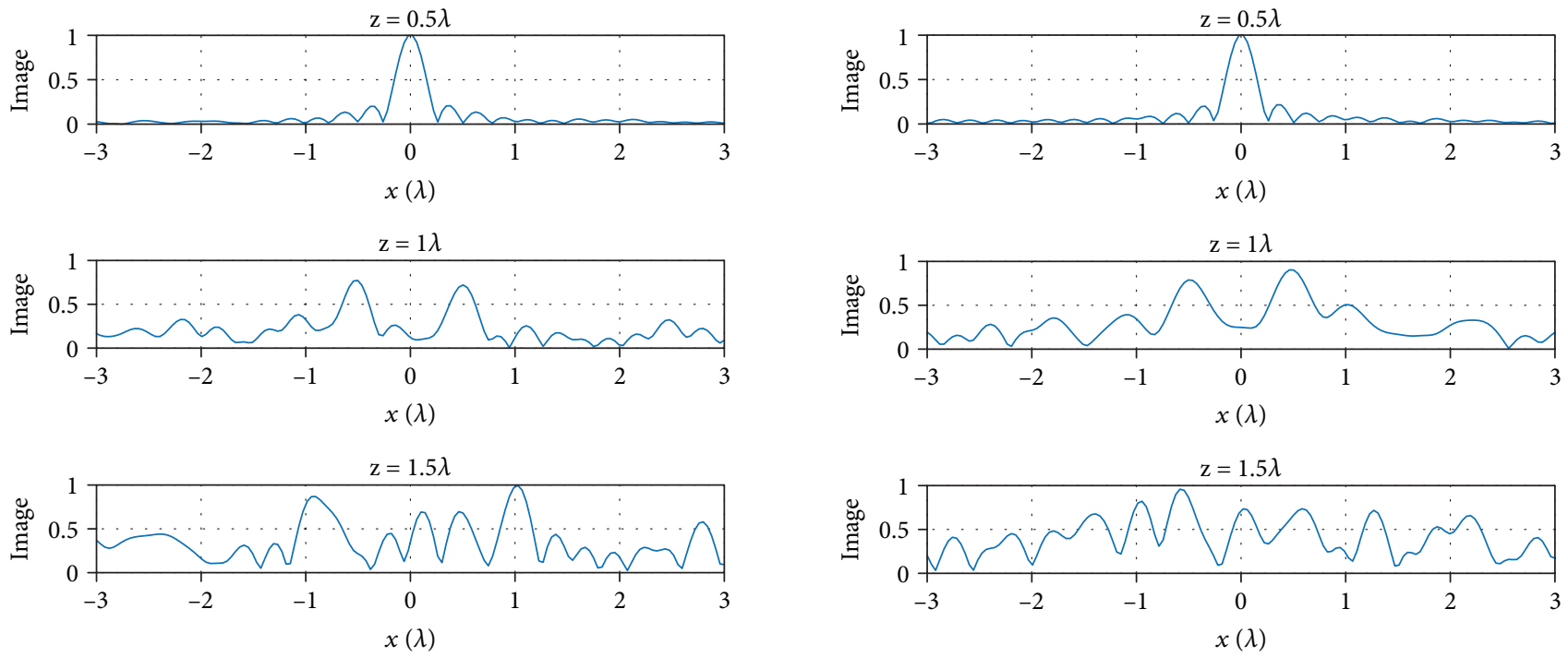

FIGURE 5: Reconstructed images when using 9 resonant dipole antennas. For this example, the parameters are $z_{1}=0.5 \lambda, z_{2}=\lambda$, $z_{3}=1.5 \lambda, d_{2}=\lambda, d_{3}=2 \lambda, s=\lambda / 8$, and $D=0.25 \lambda$. These results are with $\mathrm{SNR}=20 \mathrm{~dB}$.
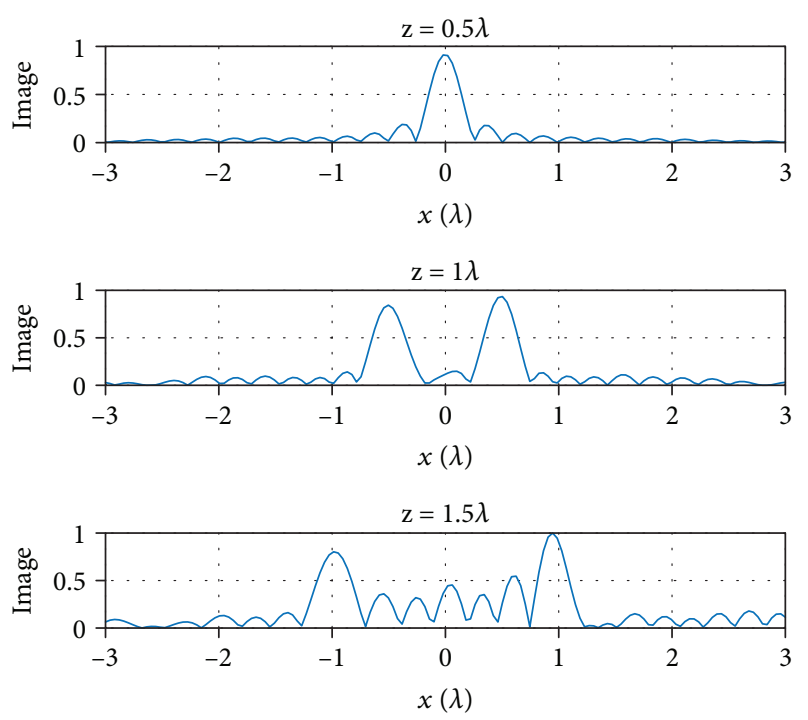

FIGURE 6: Reconstructed images when using 9 resonant dipole antennas. For this example, the parameters are $z_{1}=0.5 \lambda, z_{2}=\lambda$, $z_{3}=1.5 \lambda, d_{2}=\lambda, d_{3}=2 \lambda, s=\lambda / 8$, and $D=0.25 \lambda$. The auxiliary equations for lossless media proposed in [32] have been employed to improve the quality of the images. These results are with $\mathrm{SNR}=20 \mathrm{~dB}$.

Since unknowns $\tilde{\tilde{c}}_{i}\left(k_{x}, k_{y}\right)$ are the same for the two systems of equations in (6) and (10), these two systems of equations can be combined into a single system of equations. As discussed in [32], this significantly reduces the condition number of the matrix of coefficients for the system of equations to be solved leading to solutions with better quality.

Here, although the background and object media are lossy and with different values of conductivity, we show that constructing and using an auxiliary system of equations in

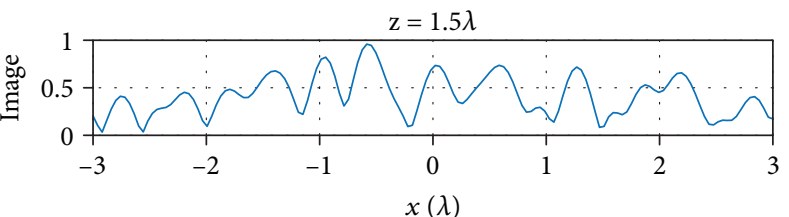

(a)
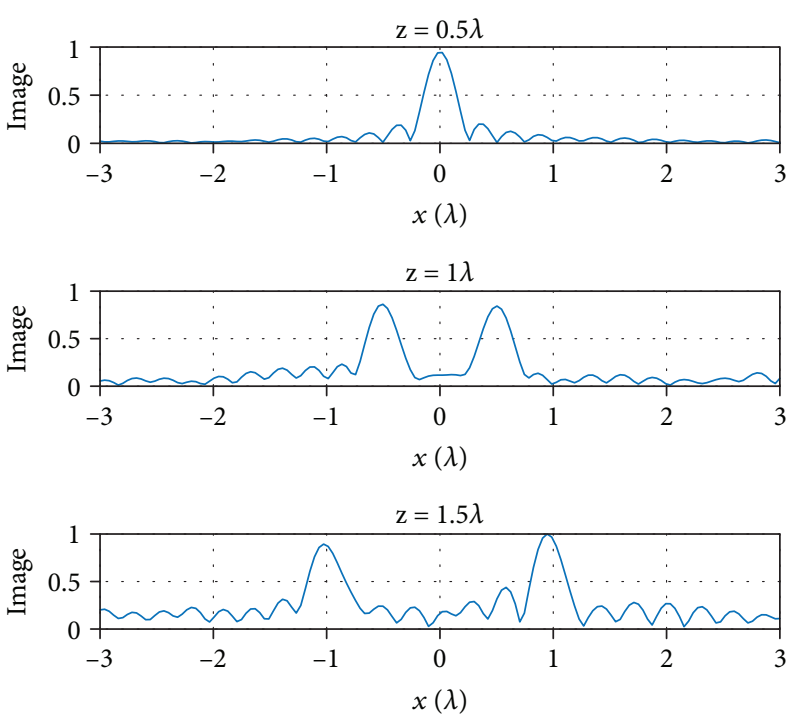

(b)

FIgURE 7: Reconstructed images when using 13 resonant dipole antennas. For this example, the parameters are $z_{1}=0.5 \lambda, z_{2}=\lambda$, $z_{3}=1.5 \lambda, d_{2}=\lambda, d_{3}=2 \lambda, s=\lambda / 8$, and (a) $D=0.125 \lambda$ (b) $D=0.5 \lambda$. These results are with $\mathrm{SNR}=20 \mathrm{~dB}$.

(10) still improves the quality of the images. It has been also mentioned in [32] that this approach still improves the quality of the images for slightly lossy media. Figure 6 shows the reconstructed images for the previous example (reduced number of antennas in the array) using such auxiliary system of equations. Comparing Figures 5 and 6 clearly shows an improvement due to the use of these auxiliary systems of equations.

To study the effect of interelement spacing $D$, again, we study the imaging setup described in the first example but with $D=0.125 \lambda$ and $D=0.5 \lambda$. Figure 7 shows the imaging results for such cases. By comparing the images in this figure with those shown in Figure 4 (for $D=0.25 \lambda$ ), it is observed that for the lowest value of $D(D=0.125 \lambda)$, the quality of 

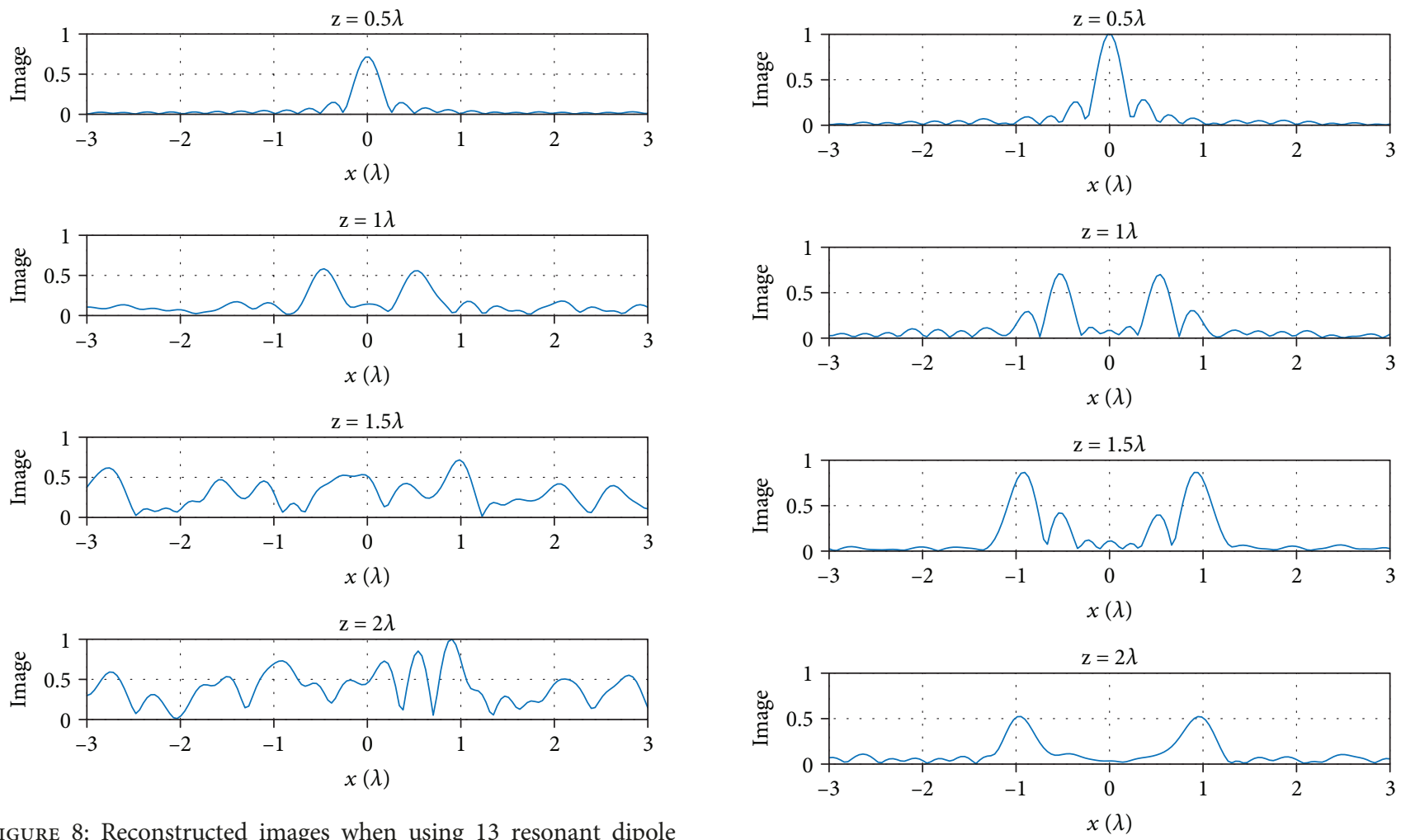

Figure 8: Reconstructed images when using 13 resonant dipole antennas. For this example, the parameters are $z_{1}=0.5 \lambda, z_{2}=\lambda$, $z_{3}=1.5 \lambda, z_{4}=2 \lambda, d_{2}=\lambda, d_{3}=2 \lambda, d_{4}=2 \lambda, s=\lambda / 8$, and $D=0.25 \lambda$. At each sampling step, the 7 th antenna element is excited and backscattered data is recorded by all antennas. These results are with $\mathrm{SNR}=20 \mathrm{~dB}$.

the images is not acceptable in particular for the image at $z=1.5 \lambda$. This is due to the fact that the rows in the system of equations in (6) get more parallel and that leads to the ill-conditioning of the system of equations. However, the results for $D=0.5 \lambda$ is as good as the results shown in Figure 4 for $D=0.25 \lambda$.

In the next step, the effect of adding an extra imaged line is studied. For this purpose, the setup described for the first example will be employed to perform imaging over four lines at $z_{1}=0.5 \lambda, z_{2}=\lambda, z_{3}=1.5 \lambda$, and $z_{4}=2 \lambda$. In other words, we add an extra imaged line at $z_{4}=2 \lambda$ compared to the first example. For implementing the imaging algorithm, the PSF corresponding to that imaged line should be also recorded beforehand, that is, by putting a small cube at $(0,0,2 \lambda)$, performing the scan by the antenna array and recoding the backscattered data. The distances between the objects are $d_{2}=\lambda$, $d_{3}=2 \lambda$, and $d_{4}=2 \lambda$. Figure 8 shows the reconstructed images. It is observed that adding one more imaged line degrades the quality of the image reconstruction results. This is due to the addition of more unknowns to the image reconstruction problem. To address this problem, we investigate the cases in which more than one antenna is excited at each sampling position. For example, Figure 9 shows the imaging results when antennas 1 and 7 are excited. These antennas are excited sequentially and in each case the backscattered waves are recorded by all the antennas. Comparing Figures 8 and 9 clearly shows significant improvement in the image

Figure 9: Reconstructed images when using 13 resonant dipole antennas. For this example, the parameters are $z_{1}=0.5 \lambda, z_{2}=\lambda$, $z_{3}=1.5 \lambda, z_{4}=2 \lambda, d_{2}=\lambda, d_{3}=2 \lambda, d_{4}=2 \lambda, s=\lambda / 8$, and $D=0.25 \lambda$. At each sampling step, the 1 st and 7 th antenna elements are excited sequentially and, for each cases, backscattered data is recorded by all antennas. These results are with $\mathrm{SNR}=20 \mathrm{~dB}$.

reconstruction results when using additional data. Thus, we proceed further to excite more antennas and study if further improvement can be achieved. In the next studied case, antennas 1, 4, and 7 are excited. Similar to the previous case, at each sampling position, these antennas are excited sequentially and for each case the backscattered waves are recorded by all the antennas. Figure 10 shows the reconstructed images for this scenario. Comparing this figure with Figures 8 and 9 shows further improvement of the quality of the reconstructed images. This again confirms the fact that by collecting more data, the quality of the images can be improved further.

In the next example, with reference to Figure 3, an imaging problem similar to the one described in the first example is solved but when the three imaged lines are at $z_{1}=\lambda / 8$ $(0.125 \lambda), z_{2}=\lambda / 4(0.25 \lambda)$, and $z_{3}=3 \lambda / 8(0.375 \lambda)$ (distance between image lines is $\lambda / 8)$. To implement the holographic imaging technique, PSF functions are recorded beforehand by sequentially putting small cubes with sides of $\lambda / 8$ at $(0,0, \lambda / 8),(0,0, \lambda / 4)$, and $(0,0,3 \lambda / 8)$ positions and for each case the backscattered waves due to these objects are scanned by the antenna array. Figure 11 shows the reconstructed images. It is observed that the objects are reconstructed well at their true positions. Then, we repeat the same imaging example but when the three imaged lines are at $z_{1}=0.1 \lambda$, $z_{2}=0.2 \lambda$, and $z_{3}=0.3 \lambda$ (distance between image lines is $\lambda / 10$ ). 

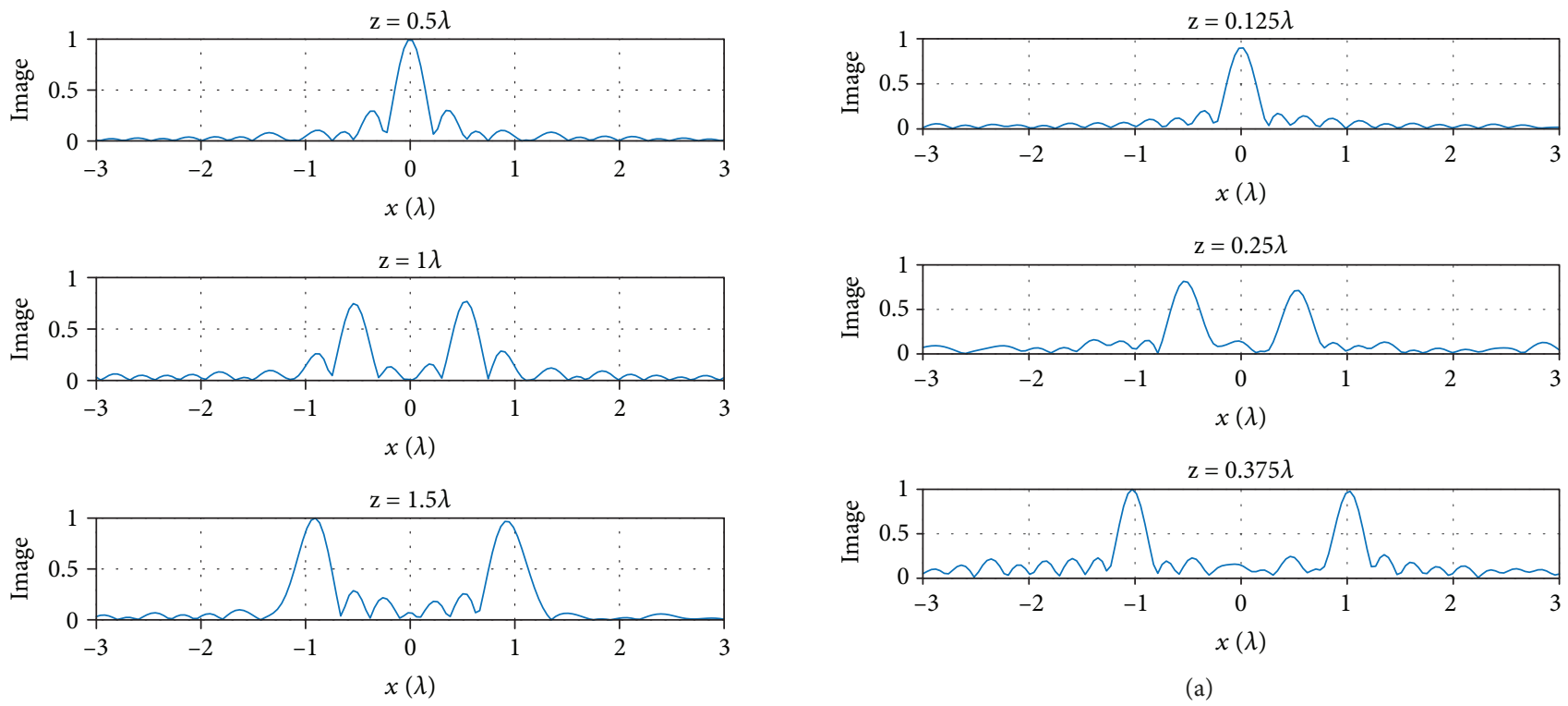

(a)
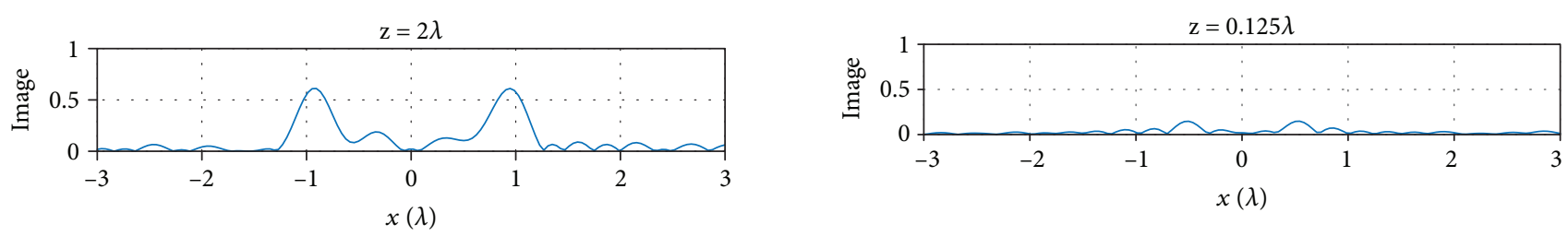

Figure 10: Reconstructed images when using 13 resonant dipole antennas. For this example, the parameters are $z_{1}=0.5 \lambda, z_{2}=\lambda$, $z_{3}=1.5 \lambda, z_{4}=2 \lambda, d_{2}=\lambda, d_{3}=2 \lambda, d_{4}=2 \lambda, s=\lambda / 8$, and $D=0.25 \lambda$. At each sampling step, the 1st, 4th, and 7 th antenna elements are excited sequentially and, for each cases, backscattered data is recorded by all antennas. These results are with $\mathrm{SNR}=20 \mathrm{~dB}$.

To implement the holographic imaging technique, PSF functions are recorded beforehand by sequentially putting small cubes with sides of $\lambda / 8$ at $(0,0,0.1 \lambda),(0,0,0.2 \lambda)$, and $(0,0,0.3 \lambda)$ positions and for each case the backscattered waves due to these objects are scanned by the antenna array. Figure 12 shows that the quality of the reconstructed images starts to degrade compared to those in Figure 11. This can be used to determine the resolution of the proposed imaging technique along the range $(z)$ direction. Based on that, the range resolution would be $\lambda / 8$ for which the imaging results have still good quality. It is worth mentioning that auxiliary systems of equations in (10) can be employed for obtaining even better resolution along the range direction.

Here, we consider the range resolution as the minimum distance along the range direction between two lines for which the reconstructed images over them clearly show the presence of the objects. Please note that, in general, the range resolution depends on the number of antennas, distance between antennas, SNR value, number of excited antennas, and number of reconstruction planes. Besides, according to our studied cases, if an object is centered at a range position that does not coincide perfectly with the range position of an imaged line, this object appears the best in the closest imaged line. Figure 11(b) shows the imaging results for a setup similar to the one presented in Figure 11(a) but when
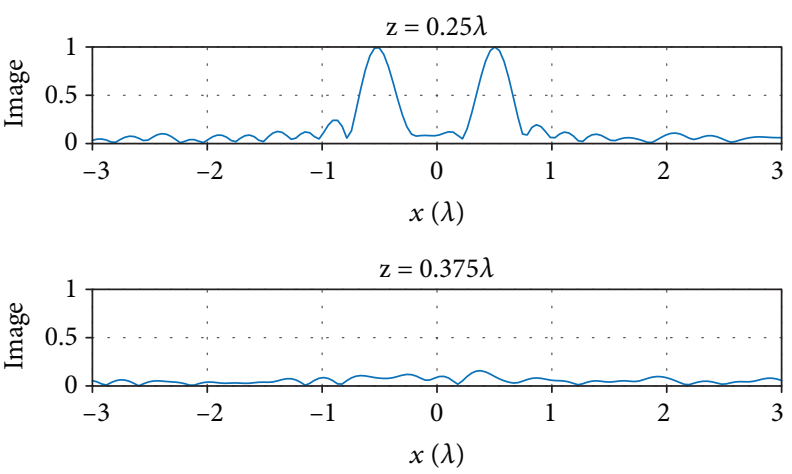

(b)

FIgURE 11: Reconstructed images when using 13 resonant dipole antennas. (a) For this example, the parameters are $z_{1}=\lambda / 8$

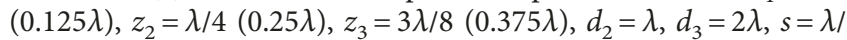
8 , and $D=0.25 \lambda$. (b) Setup similar to the one presented in Figure 11(a) but when removing the objects at $z_{1}=\lambda / 8$ and $z_{3}=3 \lambda / 8$ and shifting the objects at $z_{2}=\lambda / 4$ toward the aperture by $\lambda / 32$. In this case, these two objects will have a distance of $3 \lambda /$ 32 from the first imaged line and $\lambda / 32$ from the second imaged line. These results are with $\mathrm{SNR}=20 \mathrm{~dB}$.

removing the objects at $z_{1}=\lambda / 8$ and $z_{3}=3 \lambda / 8$ and shifting the objects at $z_{2}=\lambda / 4$ toward the aperture by $\lambda / 32$. In this case, these two objects will have a distance of $3 \lambda / 32$ from the first imaged line and $\lambda / 32$ from the second imaged line. Figure 11(b) shows the image reconstruction results in which the two objects are observed the best in the second imaged line as expected.

In the last example for the $2 \mathrm{D}$ imaging study, the effect of lower values for SNR on the image reconstruction results is studied. For this purpose, the responses used for the imaging 

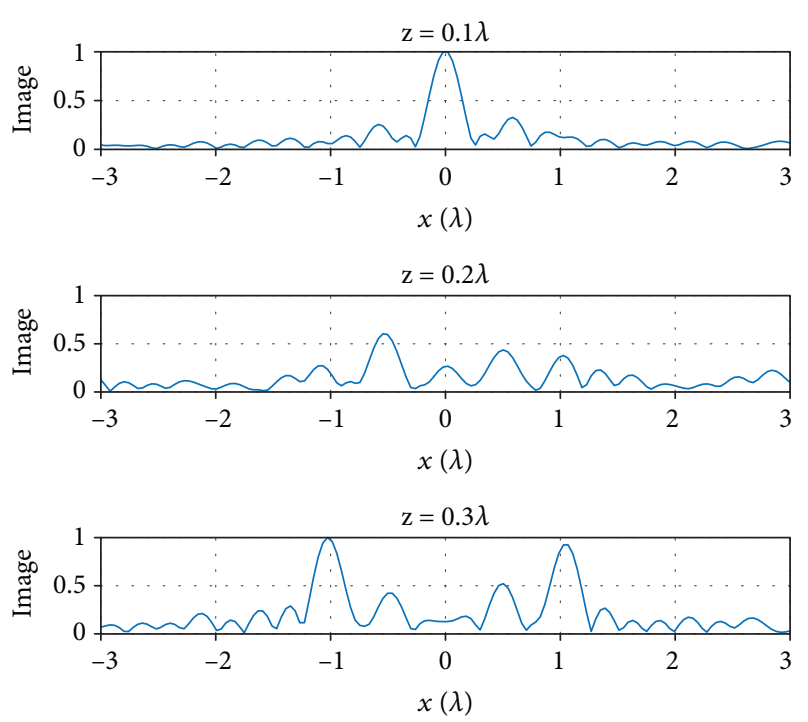

FIGURE 12: Reconstructed images when using 13 resonant dipole antennas. For this example, the parameters are $z_{1}=0.1 \lambda, z_{2}=0.2 \lambda$, $z_{3}=0.3 \lambda, d_{2}=\lambda, d_{3}=2 \lambda, s=\lambda / 8$, and $D=0.25 \lambda$. These results are with $\mathrm{SNR}=20 \mathrm{~dB}$.

example in Figure 4 are contaminated with noise with $\mathrm{SNR}=15 \mathrm{~dB}$ and $\mathrm{SNR}=10 \mathrm{~dB}$ (the results in Figure 4 are with $\mathrm{SNR}=20 \mathrm{~dB}$ ). Figures 13 and 14 show the image reconstruction results for $\mathrm{SNR}=15 \mathrm{~dB}$ and $\mathrm{SNR}=10 \mathrm{~dB}$, respectively. It is observed that while SNR of $15 \mathrm{~dB}$ still can be tolerated (the results are still acceptable in terms of quality), lower SNR values, for example $10 \mathrm{~dB}$, lead to unacceptable results. Producing images of good quality for SNR value as low as $15 \mathrm{~dB}$ shows the robustness of this imaging technique with respect to noise. It is worth mentioning that auxiliary systems of equations in (10) can be employed for obtaining good quality images even for SNR values as low as $5 \mathrm{~dB}$ according to our study.

4.2. Comparison with Multiple Frequency Results. In this section, a comparison is made between the results obtained from the antenna array using single frequency data with the results obtained from single antenna but using multiple frequency data. For this purpose, for the first example with results shown in Figure 4, we perform data acquisition with a single antenna and at 8 frequencies. This is performed once for a frequency range from $6.2 \mathrm{GHz}$ to $6.9 \mathrm{GHz}$ (with steps of $100 \mathrm{MHz}$ ) and a second time for frequency range of $5.8 \mathrm{GHz}$ to $7.2 \mathrm{GHz}$ (with steps of $200 \mathrm{MHz}$ ). Figure 15 shows the image reconstruction results for these two cases. It is observed that for the first case, a bandwidth of $0.7 \mathrm{GHz}$ is not sufficient to provide acceptable results. In the second case, when the bandwidth is doubled, the imaging results are comparable with those shown in Figure 4 for single frequency data.

4.3. Demonstration of $3 D$ Imaging Results. In this section, we demonstrate the results for 3D imaging examples. In the first example, shown in Figure 16(a), 9 objects are distributed in the $3 \mathrm{D}$ space. One object is at $z=0.5 \lambda$ plane at $(0,0,0.5 \lambda)$, four
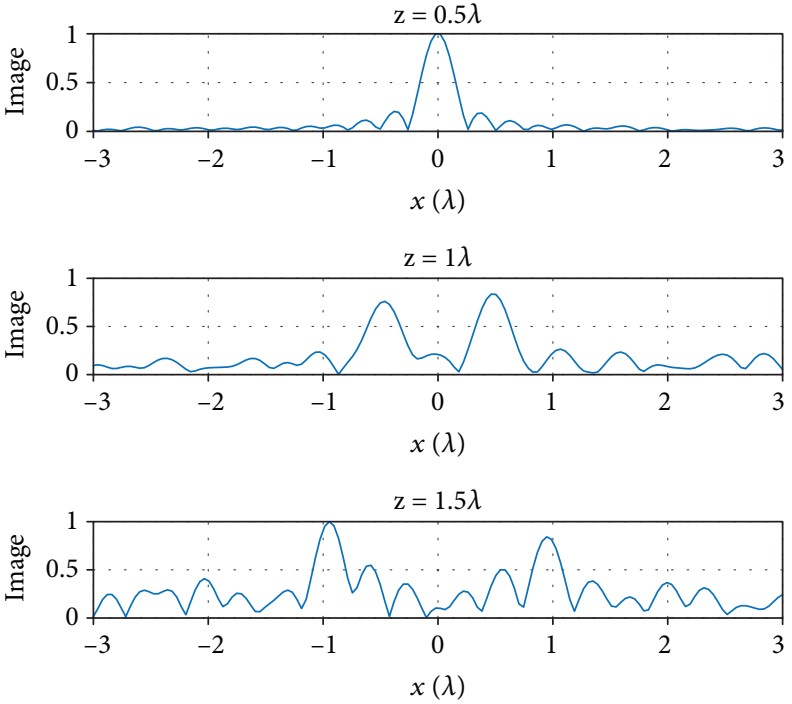

FIgURE 13: Reconstructed images when using 13 resonant dipole antennas. For this example, the parameters are $z_{1}=0.5 \lambda, z_{2}=\lambda$, $z_{3}=1.5 \lambda, d_{2}=\lambda, d_{3}=2 \lambda, s=\lambda / 8$, and $D=0.25 \lambda$. These results are with $\mathrm{SNR}=15 \mathrm{~dB}$.
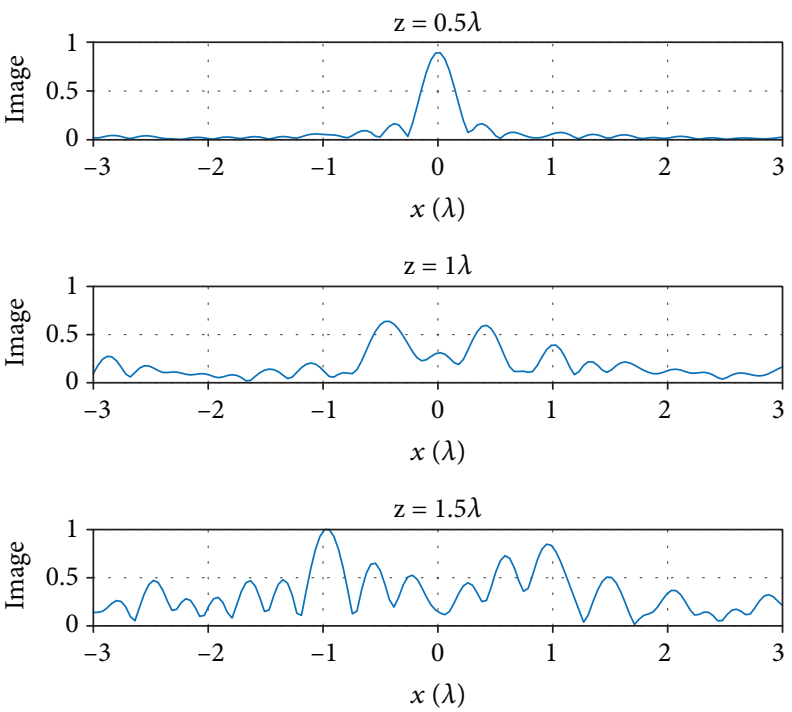

FIgURE 14: Reconstructed images when using 13 resonant dipole antennas. For this example, the parameters are $z_{1}=0.5 \lambda, z_{2}=\lambda$, $z_{3}=1.5 \lambda, d_{2}=\lambda, d_{3}=2 \lambda, s=\lambda / 8$, and $D=0.25 \lambda$. These results are with $\mathrm{SNR}=10 \mathrm{~dB}$.

objects are at $z=\lambda$ plane, at $( \pm \lambda / 2,0, \lambda)$ and $(0, \pm \lambda / 2, \lambda)$, and four objects are at $z=1.5 \lambda$ plane, at $( \pm \lambda, 0,1.5 \lambda)$ and $(0, \pm \lambda, 1.5 \lambda)$. In the second example, two $\mathrm{X}$-shape objects with an arm length of $1.2 \lambda$ and cross-section of each arm being square with a side of $\lambda / 8$ are considered. One $\mathrm{X}$ shape object is placed at $z=0.5 \lambda$ with its arms along $x$ and $y$-axis; the second object is placed at $z=1.5 \lambda$ with its arms rotated $45^{\circ}$ with respect to $x$ and $y$-axis. The properties of the background and object media are the same as in the previous examples. The number of antennas in the array and also the distance between them is similar to those reported 

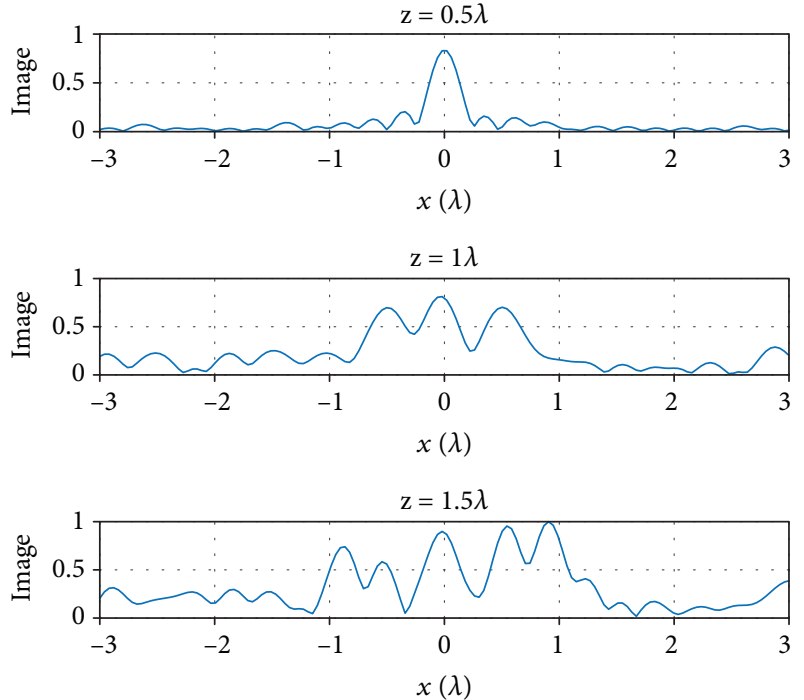

(a)
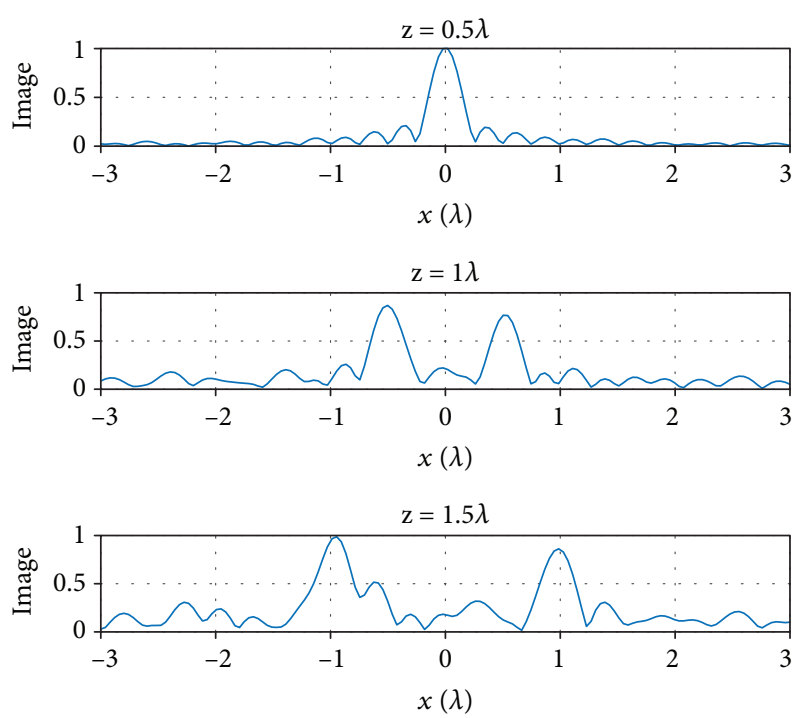

(b)

FIGURE 15: Reconstructed images when using one resonant dipole antenna and multiple frequency data: (a) using 8 frequencies with frequency range of $6.2 \mathrm{GHz}$ to $6.9 \mathrm{GHz}(100 \mathrm{MHz}$ sampling step) and (b) using 8 frequencies with frequency range of $5.8 \mathrm{GHz}$ to $7.2 \mathrm{GHz} \quad(200 \mathrm{MHz}$ sampling step). For this example, the parameters are $z_{1}=0.5 \lambda, z_{2}=\lambda, z_{3}=1.5 \lambda, d_{2}=\lambda, d_{3}=2 \lambda$, and $s=\lambda$ / 8. These results are with $\mathrm{SNR}=20 \mathrm{~dB}$.

for the first example with the results shown in Figure 4. Although here the antenna array is along the $x$-axis, in general, the array can be a $2 \mathrm{D}$ array along both $x$ and $y$ directions. To implement the holographic imaging technique, PSF functions are recorded beforehand by sequentially putting small cubes with sides of $\lambda / 8$ at $(0,0,0.5 \lambda),(0,0, \lambda)$, and $(0,0,1.5 \lambda)$ positions and for each case the backscattered waves due to these objects are scanned by the antenna array over an aperture of size $6 \lambda \times 6 \lambda$ centered at the origin. Figure 17 shows the reconstructed images. It is observed that the objects are reconstructed well at their true positions. This confirms the capability of the proposed single frequency

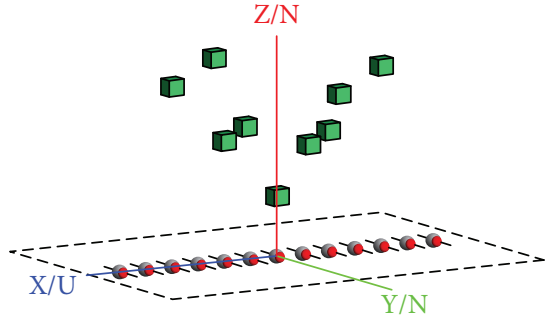

(a)

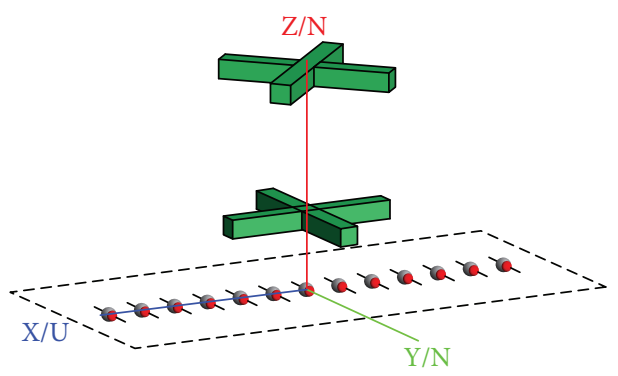

(b)

FIgURE 16: Setup in FEKO software for 3D imaging. (a) Example 1: One object is at $z=0.5 \lambda$ plane at $(0,0,0.5 \lambda)$, four objects are at $z=\lambda$ plane at $( \pm \lambda / 2,0, \lambda)$ and $(0, \pm \lambda / 2, \lambda)$, and four objects are at $z=1.5 \lambda$ plane at $( \pm \lambda, 0,1.5 \lambda)$ and $(0, \pm \lambda, 1.5 \lambda)$. Objects are square shape with side of $\lambda / 8$. (b) Example 2: Two X-shape objects with an arm length of $1.2 \lambda$ and cross-section of each arm being square with a side of $\lambda / 8$. One objects is placed at $z=0.5 \lambda$ with it arms along $x$ and $y$-axis, and the second object is placed at $z=1.5 \lambda$ with its arms rotated $45^{\circ}$ with respect to $x$ and $y$-axis.

microwave imaging setup to perform 3D imaging. Please note that, in general, the accuracy of the image reconstruction results degrades for larger objects. This is well-known for all the imaging techniques based on the use of Born approximation (e.g., see [23]).

In Figure 18, we repeat the image reconstruction results for the example 1 in Figure 16(a) while having the distance between the imaged planes as low as $\lambda / 8(\lambda / 8$ is the resolution of this imaging system obtained in Figure 11). From Figure 18, it is observed that satisfactory imaging results are obtained with the objects reconstructed well in their true positions. Small artifacts are observed on the planes not containing the objects.

\section{Conclusion}

In this paper, we proposed an approach to achieve range resolution with single frequency microwave data leading to $3 \mathrm{D}$ imaging of the inspected medium. This is in contrast to the previously proposed 3D holographic imaging techniques in which wideband data has to be acquired to perform 3D imaging.

To obtain sufficient data that allows for 3D imaging, an array of resonant antennas is employed. This array of antennas is scanned over a linear or rectangular aperture to perform $2 \mathrm{D}$ or $3 \mathrm{D}$ imaging. At each scanning step, one or more elements are excited sequentially, and the backscattered data is recorded by all the array elements. 

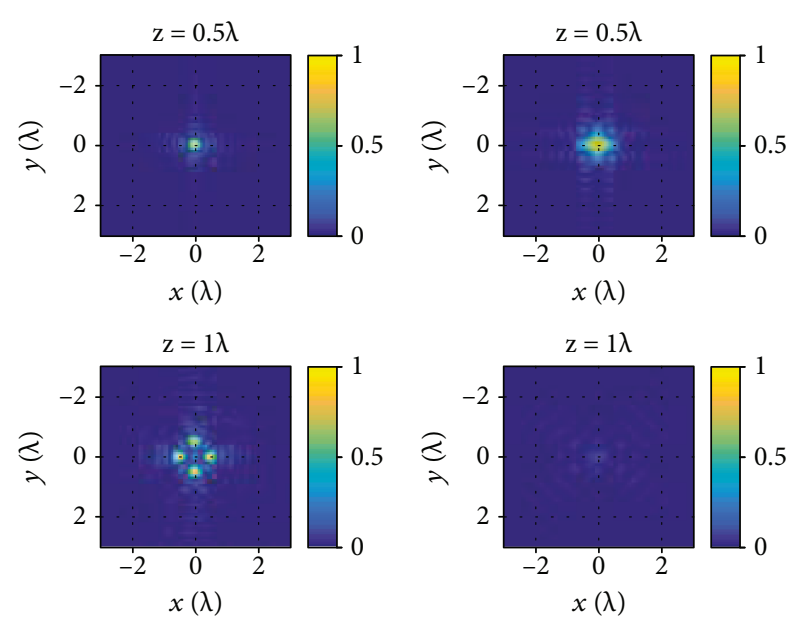

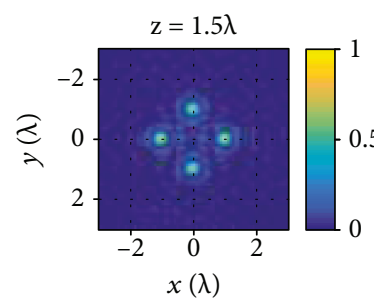

(a)

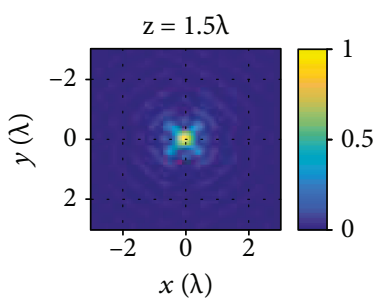

(b)
FIGURE 17: 3D imaging results for the examples shown in Figure 16 for (a) example 1 and (b) example 2. The objects can be clearly observed in the reconstructed images. These results are with $\mathrm{SNR}=30 \mathrm{~dB}$.

For easier understanding of the capabilities and limitations of this technique, the performance of this technique is first comprehensively studied in performing $2 \mathrm{D}$ imaging, that is, along one cross-range direction ( $x$-axis) and range direction ( $z$-axis). The data acquisition for this study was performed by scanning the antenna array over a linear aperture along the cross-range direction. Then, $3 \mathrm{D}$ imaging results were presented confirming the capability of the proposed technique in providing accurate 3D images.

Using the simulation data allowed for a comprehensive investigation of the limitations of the technique with respect to the number of antennas in the array, number of imaged lines (planes), number of excitations, distance between the antennas in the array, and the effect of noise. Besides, contaminating the simulation data with noise provided a realistic evaluation of the performance of the proposed technique.

The cross-range resolution for the proposed technique can be obtained from conventional expressions used for holographic imaging. However, the quality of the images that ultimately can be used for evaluating both the cross-range resolution and range resolution depends on how accurate the corresponding systems of equations can be solved. This, in turn, depends on the number of antennas, distance between imaged lines (planes), number of imaged lines (planes), amount of noise, spacing between the antennas in the array, and so on. In this study, a resolution of $\lambda / 8$ was
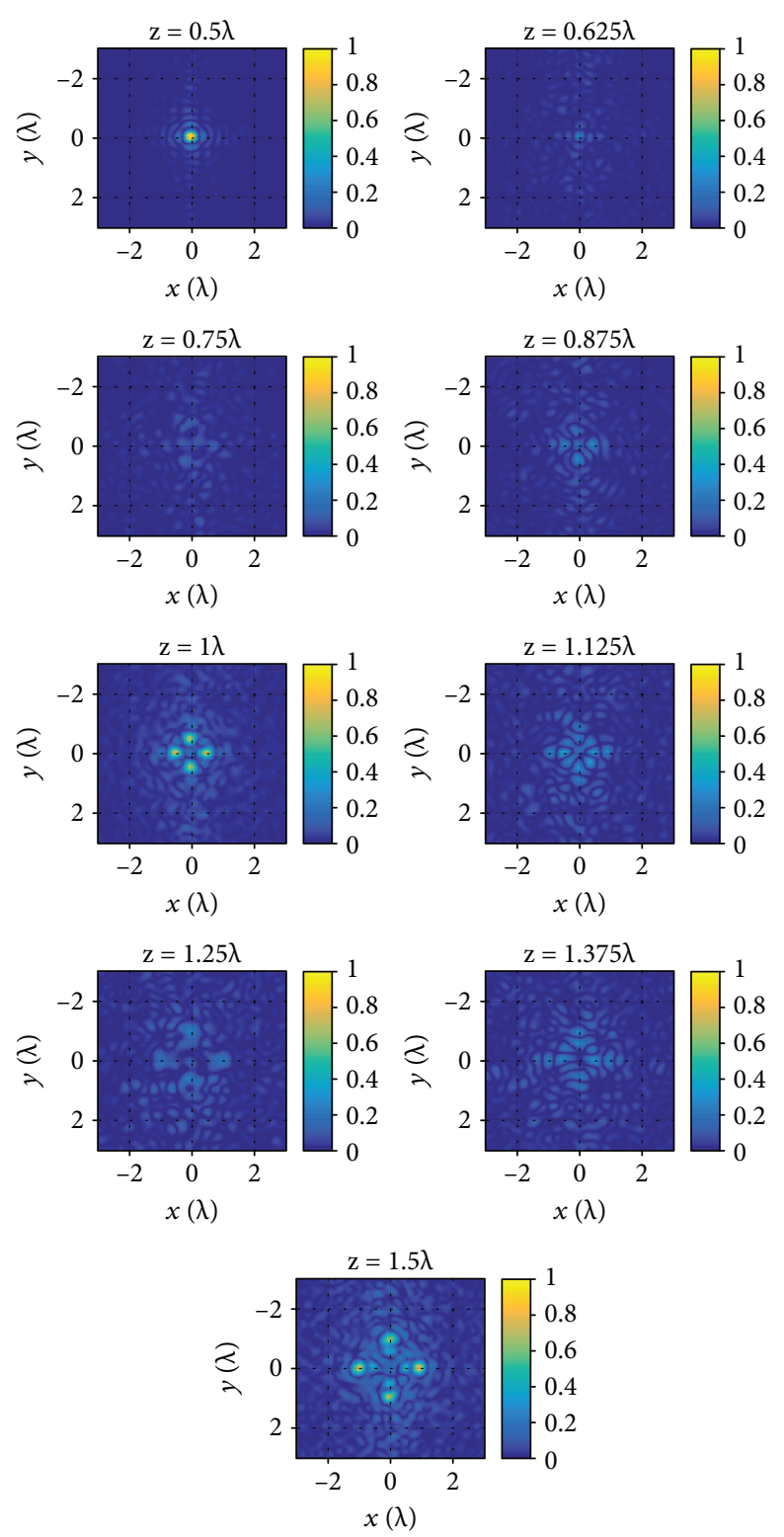

FIgURE 18: 3D imaging results for the example 1 shown in Figure 16(a). The number of imaged planes has been increased compared to the results in Figure 17(a). The distance between the planes is $\lambda / 8$ (the resolution determined by the $2 \mathrm{D}$ study). These results are with $\mathrm{SNR}=30 \mathrm{~dB}$.

demonstrated along the range for the studied setup which is excellent for many applications such as biomedical imaging.

We emphasize that in the results presented in this manuscript we have included the effect of noise on the complexvalued scattered field. In particular, in Figures 13 and 14, we have studied the threshold for SNR (10 dB or so) above which the technique performs well. In practice, the source of noise can be any electronic or mechanical issue such as problems in source coherence and ripples in mechanical scanning of the sensor. Comparing these results with those in [23] (where noise study has been performed for wideband near-field holography) shows that the single frequency holography proposed here would be more vulnerable to 
the noise. Although a threshold of $10 \mathrm{~dB}$ or so is still very promising in the proposed work; in [23], the wideband holographic imaging provides satisfactory imaging results for SNR as low as $-10 \mathrm{~dB}$.

As a final note, using single frequency data in the proposed technique allows for employing data acquisition techniques such as modulated scatterer technique (MST) [33] or employing resonant antennas or probes which are more sensitive, for example, in microwave or millimeter wave microscopy applications (e.g., see [34]).

\section{Data Availability}

The data used in this manuscript was generated by FEKO simulations software as mentioned in the paper. All the dimensions and material information have been provided in the paper so that a reader can repeat the work.

\section{Conflicts of Interest}

The authors declare that they have no conflicts of interest.

\section{References}

[1] D. Gabor, "A new microscopic principle," Nature, vol. 161, no. 4098, pp. 777-778, 1948.

[2] D. Gabor, "Microscopy by reconstructed wave-fronts," Proceedings of the Royal Society A: Mathematical, Physical and Engineering Sciences, vol. 197, no. 1051, pp. 454-487, 1949.

[3] E. N. Leith and J. Upatnieks, "Reconstructed wavefronts and communication theory," Journal of the Optical Society of America, vol. 52, no. 10, pp. 1123-1130, 1962.

[4] E. N. Leith and J. Upatnieks, "Wavefront reconstruction with continuous-tone objects," Journal of the Optical Society of America, vol. 53, no. 12, pp. 1377-1381, 1963.

[5] B. P. Hildebrand and B. B. Brenden, An Introduction to Acoustical Holography, Plenum Press, 1972.

[6] R. P. Dooley, "X-band holography," Proceedings of the IEEE, vol. 53, no. 11, pp. 1733-1735, 1965.

[7] D. E. Duffy, "Optical reconstruction from microwave holograms," Journal of the Optical Society of America, vol. 56, no. 6, pp. 832-832, 1966.

[8] G. Tricoles and E. L. Rope, "Reconstructions of visible images from reduced-scale replicas of microwave holograms," Journal of the Optical Society of America, vol. 57, no. 1, pp. 97-99, 1967.

[9] W. E. Kock, "Stationary coherent (hologram) radar and sonar," Proceedings of the IEEE, vol. 56, no. 12, pp. 21802181, 1968.

[10] W. E. Kock, "Microwave holography," in Engineering Applications of Lasers and Holography, pp. 179-223, Springer, Boston, MA, USA, 1975.

[11] E. N. Leith, "Quasi-holographic techniques in the microwave region," Proceedings of the IEEE, vol. 59, no. 9, pp. 1305-s-1318, 1971.

[12] N. H. Farhat, "Microwave holography and coherent tomography," in Medical Applications of Microwave Imaging, L. E. Larsen and J. H. Jacobi, Eds., pp. 66-81, IEEE PRESS, 1986.
[13] G. Tricoles and N. H. Farhat, "Microwave holography: applications and techniques," Proceedings of the IEEE, vol. 65, no. 1, pp. 108-121, 1977.

[14] M. Elsdon, O. Yurduseven, and D. Smith, "Early stage breast cancer detection using indirect microwave holography," Progress In Electromagnetics Research, vol. 143, pp. 405-419, 2013.

[15] O. Yurduseven, D. Smith, B. Livingstone, V. Schejbal, and Z. You, "Investigations of resolution limits for indirect microwave holographic imaging," International Journal of RF and Microwave Computer-Aided Engineering, vol. 23, no. 4, pp. 410-416, 2013.

[16] O. Yurduseven, "Indirect microwave holographic imaging of concealed ordnance for airport security imaging systems," Progress In Electromagnetics Research, vol. 146, pp. 7-13, 2014.

[17] D. M. Sheen, D. L. McMakin, and T. E. Hall, "Threedimensional millimeter-wave imaging for concealed weapon detection," IEEE Transactions on Microwave Theory and Techniques, vol. 49, no. 9, pp. 1581-1592, 2001.

[18] D. Sheen, D. McMakin, and T. Hall, "Near-field threedimensional radar imaging techniques and applications," Applied Optics, vol. 49, no. 19, pp. E83-E93, 2010.

[19] M. Soumekh, "A system model and inversion for synthetic aperture radar imaging," IEEE Transactions on Image Processing, vol. 1, no. 1, pp. 64-76, 1992.

[20] D. McMakin, D. Sheen, T. Hall, J. Tedeschi, and A. M. Jones, "New improvements to millimeter-wave body scanners," in Proceedings of 3DBODY.TECH 2017 - 8th International Conference and Exhibition on 3D Body Scanning and Processing Technologies, Montreal QC, Canada, October 2017.

[21] B. G. Braatz, A. M. Jones, J. R. Tedeschi et al., "Cleareye inground and in-concrete DIV inspections: FY11 final report," Tech. Rep. 21053, Pacific Northwest National Laboratory (PNNL), Richland, WA USA, 2012.

[22] A. Zhuravlev, S. Ivashov, V. Razevig, and I. Vasiliev, "Shallow depth subsurface imaging with microwave holography," in Proceedings Volume 9072, Detection and Sensing of Mines, Explosive Objects, and Obscured Targets, Baltimore, MD, USA, May 2014.

[23] R. K. Amineh, M. Ravan, A. Khalatpour, and N. K. Nikolova, "Three-dimensional near-field microwave holography using reflected and transmitted signals," IEEE Transactions on Antennas and Propagation, vol. 59, no. 12, pp. 4777-4789, 2011.

[24] M. Ravan, R. K. Amineh, and N. K. Nikolova, "Two-dimensional near-field microwave holography," Inverse Problems, vol. 26, no. 5, article 055011, 2010.

[25] R. K. Amineh, A. Khalatpour, H. Xu, Y. Baskharoun, and N. K. Nikolova, "Three-dimensional near-field microwave holography for tissue imaging," International Journal of Biomedical Imaging, vol. 2012, Article ID 291494, 11 pages, 2012.

[26] R. K. Amineh, M. Ravan, J. McCombe, and N. K. Nikolova, "Three-dimensional microwave holographic imaging employing forward-scattered waves only," International Journal of Antennas and Propagation, vol. 2013, 15 pages, 2013.

[27] R. K. Amineh, J. J. McCombe, A. Khalatpour, and N. K. Nikolova, "Microwave holography using point-spread functions measured with calibration objects," IEEE Transactions on Instrumentation and Measurement, vol. 64, no. 2, pp. 403-417, 2015. 
[28] L. Wang, R. Simpkin, and A. M. Al-Jumaily, "Threedimensional far-field holographic microwave imaging: an experimental investigation of dielectric object," Progress in Electromagnetics Research B, vol. 61, pp. 169-184, 2014.

[29] W. Chew, Waves and Fields in Inhomogeneous Media, IEEE Press, Piscataway, NJ, USA, 1995.

[30] A. N. Tikhonov and V. Y. Arsenin, Solutions of Ill-Posed Problems, Wiley, New York, NY, USA, 1977.

[31] "FEKO software," https://altairhyperworks.com/product/ FEKO.

[32] C.-H. Tsai, J. Chang, L.-Y. O. Yang, and S.-Y. Chen, "3-D microwave holographic imaging with probe and phase compensations," IEEE Transactions on Antennas and Propagation, vol. 66, no. 1, pp. 368-380, 2018.

[33] J. C. Bolomey and F. E. Gardiol, Engineering Applications of the Modulated Scattering Technique, Artech House Publishers, 2001.

[34] A. Imtiaz, T. M. Wallis, and P. Kabos, "Near-field scanning microwave microscopy: an emerging research tool for nanoscale metrology," IEEE Microwave Magazine, vol. 15, no. 1, pp. 52-64, 2014. 


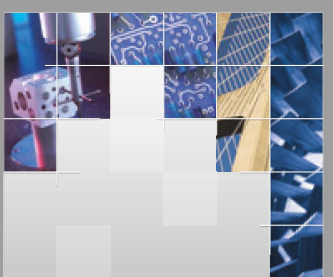

\section{Enfincering}
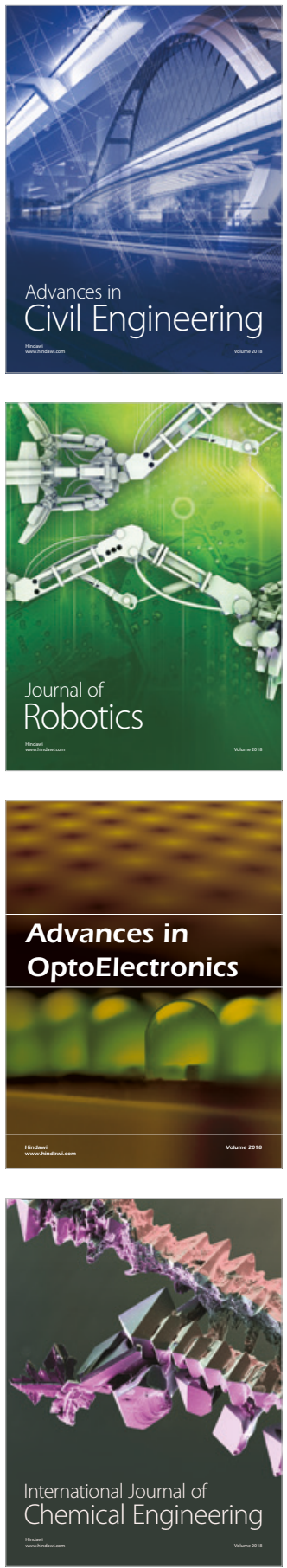

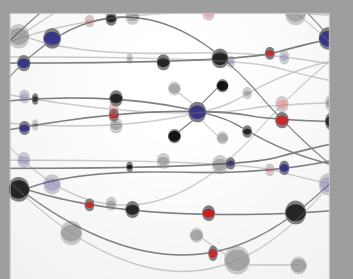

\section{Rotating \\ Machinery}

The Scientific World Journal

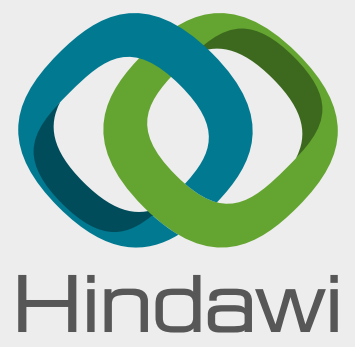

Submit your manuscripts at

www.hindawi.com
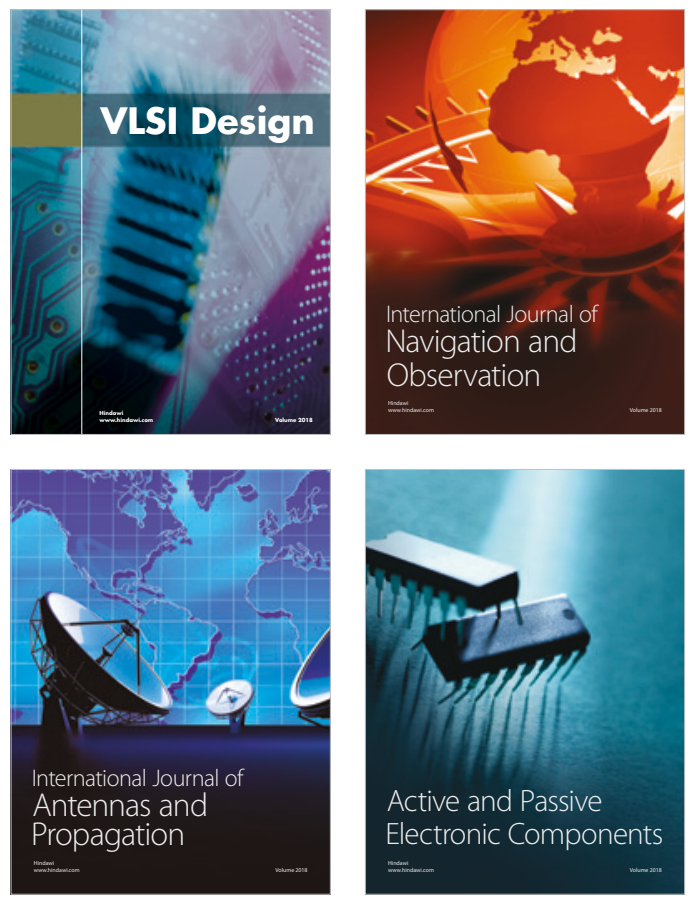
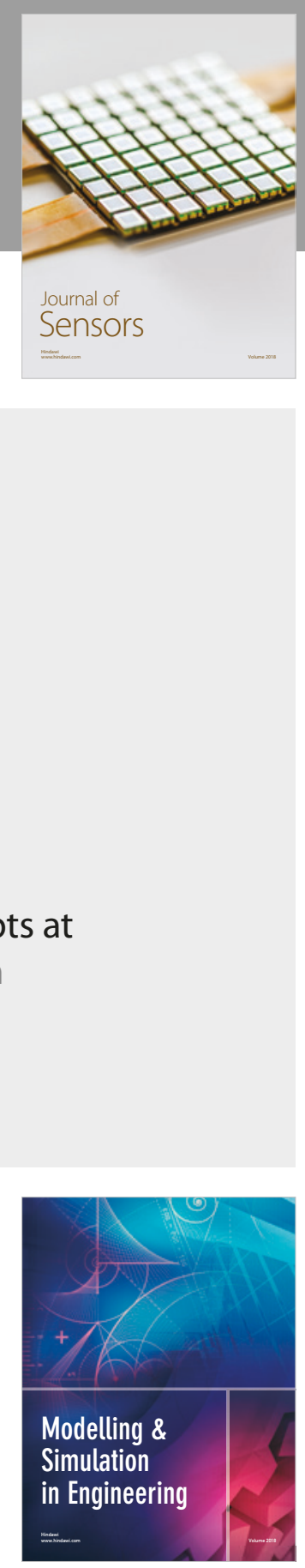

\section{Advances \\ Multimedia}
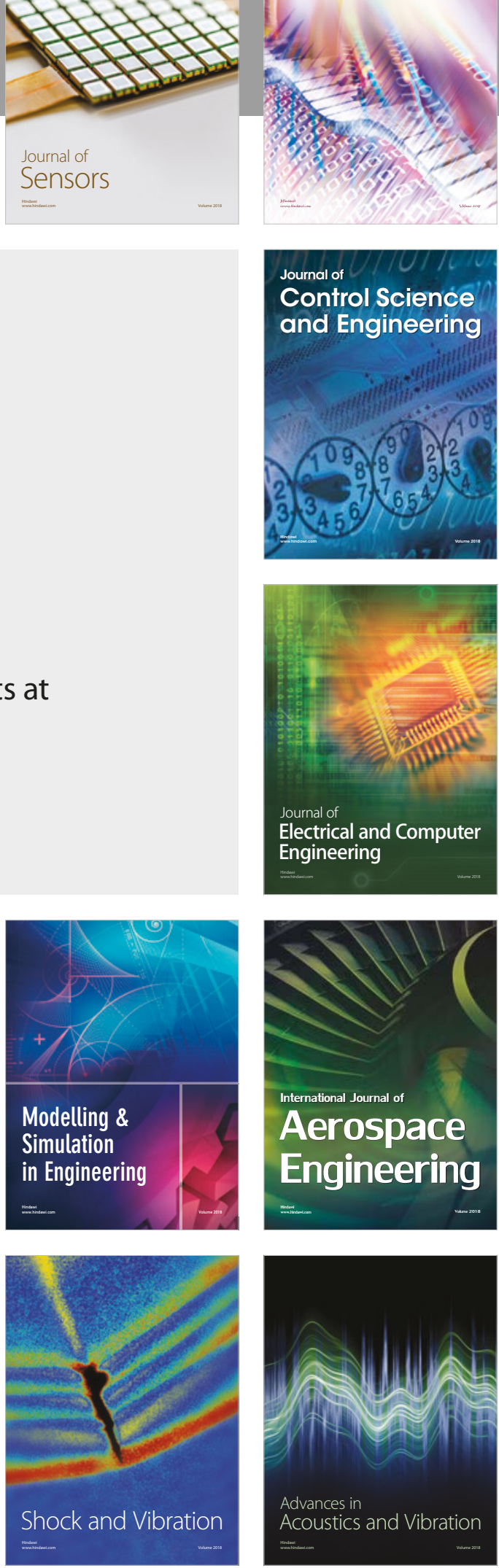